\title{
New Reconfiguration Method for Improving Voltage Profile of Distribution Networks
}

\author{
S. Aruul Vizhiy \\ Associate professor, Department of EEE \\ CK College of Engineering and Technology \\ Cuddalore-607003, Tamil Nadu, India
}

\author{
R.K. Santhi \\ Professor \\ Electrical Engineering, Annamalai University \\ India
}

\begin{abstract}
Network reconfiguration aims to minimize network real power loss through rearranging the status of open switches. The consumers of the distribution networks need a better voltage profile for efficient operation of various gadgets. This paper thus attempts to develop a new reconfiguration algorithm with an objective of improving the voltage profile of the distribution network without incurring any additional cost for installation of capacitors and tap-changing transformers. The algorithm uses a nature-inspired biogeography based optimization (BBO) that searches for optimal solution through the migration and mutation operators. Test results on a 33 and 69-node distribution networks reveal the superiority of the developed method.
\end{abstract}

\section{General Terms}

Optimization, evolutionary algorithms

\section{Keywords}

radial distribution networks, network reconfiguration, biogeography based optimization.

$\begin{array}{ll}\text { Nomenclature } & \\ \text { BBO } & \begin{array}{l}\text { biogeography based optimization } \\ \text { branch-to-node matrix that describes the } \\ \text { topological structure of the distribution } \\ \text { network }\end{array} \\ \text { GA } & \begin{array}{l}\text { genetic algorithm } \\ \text { habitat suitability index }\end{array} \\ H S I & i^{\text {th }} \text { habitat } \\ h_{i} & \text { vector of load currents } \\ {\left[I_{L}\right]} & \text { vector of branch currents } \\ {\left[i_{b}\right]} & \text { equivalent load current at node- } m \\ I_{L, m} & \text { maximum number of iterations for } \\ I t e r^{\text {max }} & \text { convergence check } \\ n n & \text { number of nodes } \\ n b & \text { number of branches } \\ n e h & \text { number of elite habitats } \\ \text { PSO } & \text { particle swarm optimization } \\ P^{\text {mod }} & \text { habitat modification probability } \\ P_{m} & \text { mutation probability } \\ P_{L-m}+j Q_{L-m} & \text { real and reactive power load at node- } m \\ r_{l}+j x_{l} & \text { resistance and reactance of branch- } l \\ S^{\max } & \text { maximum species count } \\ S I V & \text { suitability index variable } \\ O S S_{j} & \text { binary variable that represents the topological } \\ & \text { status of } j \text {-th branch. It equals ' } 1 \text { ', if the } \\ \text { tie/sectionalizing switch is closed, else its } & \text { value is set as '0' }\end{array}$

$\begin{array}{ll}\text { VPI } & \text { voltage profile improvement } \\ V_{m} \angle \delta_{m} & \text { voltage at node-m } \\ V_{o} \angle \delta_{o} & \text { voltage at source node } \\ \text { LVM } & \text { lowest node voltage seen in the network } \\ \text { NVD } & \text { net voltage deviations } \\ {\left[v_{b}\right]} & \text { vector of branch voltage drops } \\ {[z]} & \text { diagonal matrix containing the self- } \\ \mathfrak{R} & \text { impedances of all the branches } \\ \lambda & \text { a set of branches, whose current flow exceed } \\ \mu & \text { immigration rate } \\ w_{b} \text { and } w_{c} & \text { emigration rate }\end{array}$

\section{INTRODUCTION}

Network reconfiguration is a process of altering the topological structures of the distribution feeders by changing the open/close status of the sectionalizing and tie switches. During normal operating conditions, networks are reconfigured to reduce system real power loss, achieve load balancing and relieve network overloads. The network reconfiguration problem was first solved for loss reduction using branch-and-bound-type optimization technique by Merlin and Back in the year 1975 [1]. In that method, all network switches are initially closed to form a meshed network and then the switches are successively opened to restore radial configuration. Deterministic mathematical approaches involving Benders decomposition [2], and mixedinteger programming $[3,4]$ have been proposed. A switch exchange method has been outlined in [5] and further modified in [6]. A heuristic type algorithm to find the tieswitch position in each loop to reduce the loss has been proposed [7]. These algorithms start with a radial configuration of the network and modify the network configuration using heuristic formulas in order to reduce the network loss. They are usually fast but may not achieve the optimal configuration. Therefore, metaheuristic algorithms such as hyper-cube ant colony optimization [8], bacterial foraging optimization algorithm [9], particles swarm optimization [10], artificial immune systems [11], adaptive imperialist competitive algorithm [12], and genetic algorithms [13] have been gradually applied for reconfiguration to reduce the network loss. Metaheuristic optimization methods can solve the reconfiguration problem without any or fewer restrictions on the shape of the cost function curves due to their ability to seek the global optimal solution. Moreover, these algorithms do not depend on the first and second differentials of the objective function.

Recently, a Biogeography-Based Optimization (BBO), a population based meta-heuristic optimization technique 
sharing information between candidate solutions based on their fitness values, has been suggested for solving optimization problems by Simon [14]. It has been applied to a variety of power system optimization problems [15-18] and found to yield satisfactory results.

The objectives of traditional reconfiguration schemes are to reduce network real power loss, achieve load balancing and relieve network overloads. These objectives are built from the view point of service providers, but the consumers requires quality power supply with a voltage nearer to nominal voltage. This paper thus aims to develop a new reconfiguration scheme that minimizes the net deviation between the node voltages and the nominal voltage value, using BBO. The method is tested on 33- and 69-node radial networks and the results are presented.

\section{BIOGEOGRAPHY BASED OPTIMIZATION}

BBO, based on the concept of biogeography, is a stochastic optimization technique for solving multimodal optimization problems [14]. In BBO, a solution is represented by a habitat-

$i$ consisting of solution features named Suitability Index Variables ( $S I V)$, which are represented by real numbers. It is represented for a problem with $n d$ decision variables as

$h_{i}=\left[S I V_{i, 1}, S I V_{i, 2}, S I V_{i, 3}, \cdots, S I V_{i, n d}\right]$

The suitability of sustaining larger number of species of a habitat- $i$ can be modeled as a fitness measure referred to Habitat Suitability Index ( $H S I)$ as

$$
H S I_{i}=f\left(h_{i}\right)=f\left(S I V_{i, 1}, S I V_{i, 2}, S I V_{i, 3}, \cdots, S I V_{i, n d}\right)
$$

High HSI represents a better quality solution and low HSI denotes an inferior solution. The aim is to find optimal solution in terms of SIV that maximizes the HSI .

Each habitat is characterized by its own immigration rate $\lambda$ and emigration rate $\mu$. A good solution enjoys a higher $\mu$ and lower $\lambda$ and vice-versa. The immigration and emigration rates are the functions of the number of species in the habitat and defined for a habitat containing $k$-species as

$$
\begin{aligned}
& \mu_{k}=E^{\max }\left(\frac{k}{n}\right) \\
& \lambda_{k}=I^{\max }\left(1-\frac{k}{n}\right)
\end{aligned}
$$

When $E^{\max }=I^{\max }$, the immigration and emigration rates can be related as

$$
\lambda_{k}+\mu_{k}=E^{\max }
$$

A population of candidate solutions is represented as a vector of habitats similar to any other evolutionary algorithm. The features between the habitats are shared through migration operation, which is probabilistically controlled through habitat modification probability, $P^{\text {mod }}$. If a habitat $h_{i}$ in the population is selected for modification, then its $\lambda$ is used to probabilistically decide whether or not to modify each SIV in that habitat. The $\mu$ of other solutions are thereafter used to select which of the habitats in the population shall migrate randomly chosen $S I V s$ to the selected solution $h_{i}$.

The cataclysmic events that drastically change the HSI of a habitat is represented by mutation of SIVs. The mutation operation modifies a habitat's SIV randomly based on mutation rate $P_{m}$ and tends to increase diversity among the population, avoids the dominance of highly probable solutions and provides a chance of improving the low HSI solutions. Mutation rate of each solution set can be calculated in terms of species count probability using the following equation:

$$
P_{m}=m^{\max }\left(\frac{1-P^{k}}{P^{\max }}\right)
$$

\section{PROBLEM FORMULATION}

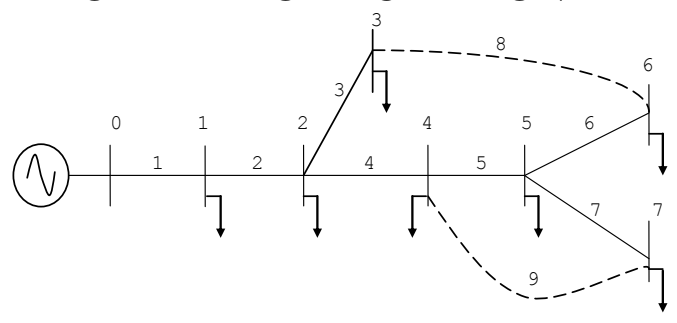

Fig. 1 Eight node example network

\subsection{Distribution Power Flow}

The equivalent load current at node- $m\left(I_{L, m}\right)$ of the distribution network can be computed from the specified real and reactive power loads $\left(P_{L-m}+j Q_{L-m}\right)$ as

$$
I_{L, m}=\left(\frac{P_{L-m}+j Q_{L-m}}{V_{m} \angle \delta_{m}}\right)^{*} \quad m=1,2, \cdots n n
$$

The branch currents in terms of equivalent load currents of all the nodes of the example network of Fig. 1 can be written as

$$
\left[\begin{array}{l}
i_{b, 1} \\
i_{b, 2} \\
i_{b, 3} \\
i_{b, 4} \\
i_{b, 5} \\
i_{b, 6} \\
i_{b, 7} \\
i_{b, 8} \\
i_{b, 9}
\end{array}\right]=\left[\begin{array}{lllllll}
1 & 1 & 1 & 1 & 1 & 1 & 1 \\
0 & 1 & 1 & 1 & 1 & 1 & 1 \\
0 & 0 & 1 & 0 & 0 & 0 & 0 \\
0 & 0 & 0 & 1 & 1 & 1 & 1 \\
0 & 0 & 0 & 0 & 1 & 1 & 1 \\
0 & 0 & 0 & 0 & 0 & 1 & 0 \\
0 & 0 & 0 & 0 & 0 & 0 & 1 \\
0 & 0 & 0 & 0 & 0 & 0 & 0 \\
0 & 0 & 0 & 0 & 0 & 0 & 0
\end{array}\right]\left[\begin{array}{c}
I_{L, 1} \\
I_{L, 2} \\
I_{L, 3} \\
I_{L, 4} \\
I_{L, 5} \\
I_{L, 6} \\
I_{L, 7}
\end{array}\right]
$$

That is,

$$
\left[i_{b}\right]=[C]\left[I_{L}\right]
$$

The voltage drop across all branches can be computed from the relation

$$
\left[v_{b}\right]=[z]\left[i_{b}\right]
$$

Similar to Eq. (3), the node voltages can be written in terms of branch voltages by the relation

$V_{i} \angle \delta_{i}=V_{o} \angle \delta_{o}-\sum_{j=1}^{n b} T_{j} C_{j i} v_{b, j}, \quad i=1,2, \cdots, n n$ 


\subsection{Objective Function}

The objective of the reconfiguration problem is to minimize the net voltage deviations (NVD) and can be formulated as

$$
\text { Minimize } N V D=\sum_{j=1}^{n n}\left|V_{j}-1.0\right|
$$

The above objective can be calculated from the node voltages, obtained through iteratively solving the distribution power flow algorithm of Eqs. 7, 9, 10 and 11 for a given load data, and status of tie and sectionalizing switches.

\subsection{Radial Topology Constraint}

The switching operation may sometimes make certain nodes disconnected from the live network, thereby making the configuration not radial. A check is therefore made for the status of tie and sectionalizing switches as to whether the resulting configuration is radial. If the network is radial, the entries in the first row of $C$ matrix are binary ones. This can be imposed as a constraint in the reconfigurations problem as

$$
\left|\sum_{j=1}^{n n} C_{1, j}-n n\right|=0
$$

If the above constraint is not satisfied, the network is either not radial or a group of loads is disconnected from service.

\subsection{Line Flow Constraint}

The resulting flows in the lines should not exceed their respective thermal limits. It is represented by a constraint

$$
\left|i_{j}\right| \leq i_{j}^{\max }
$$

\section{PROPOSED METHOD}

The aim of this section is to develop a BBO based reconfiguration method (BRM) for improving the voltage profile (VP). It involves representation of problem variables and formation of a HSI function.

The decision variable in BRM is the open-switch numbers. Each habitat of the $\mathrm{BBO}$ is therefore represented in vector form to denote the open-switches as

$$
h=\left[O S_{1}, O S_{2}, \cdots \cdots O S_{n}\right]
$$

The BBO generates real numbers and hence to obtain integer values for open-switches, the real numbers are rounded off to the nearest integer values. The HSI function can be built from the problem objective function and radial topology constraint as

\section{Maximize}

$$
H S I=\frac{1}{1+\left(N V D+w_{b} \sum_{i \in \mathfrak{R}}\left(\left|i_{j}\right|-i_{j}^{\max }\right)^{2}+w_{c}\left|\sum_{j=1}^{n n} C_{1, j}-n n\right|\right)}
$$

An initial population of habitats is obtained by generating random values within their respective limits to every individual in the population. For each habitat in the population, the NVD is calculated by the procedure discussed in section-3, after altering the network topology according to the status of open-switches, and then the HSI is computed. The migration and mutation operations are performed for non- elite habitats with a view of maximizing the HSI. This process is continued till convergence. The algorithmic steps of the proposed BRM are summarized below.

1. Read reconfiguration problem data

2. Choose $\mathrm{BBO}$ parameters such as $n h$, neh, $I^{\max }, E^{\max }$, $P^{\text {mod }}$, Iter $^{\max }$

3. Randomly generate SIVs to form the population.

4. For each habitat,

- Obtain the details of open-switches from the SIV values

- Alter the distribution network according to the details of open-switches

- Carryout distribution power flow and then compute NVD using Eq. (12)

- $\quad$ Evaluate HSI using Eq. (16)

5. Identify neh elite habitats having highest $H S I$ and retain them as it is without making any modifications.

6. Perform migration probabilistically on those SIVS of non-elite habitats.

7. Perform mutation operation probabilistically on those non-elite habitats

8. Check for convergence. If converged, go to next step. Else, go to step (4).

9. The open-switches in the best habitat represent the optimal solution.

10. Stop.

\section{NUMERICAL RESULTS}

The BRM is tested on two standard distribution networks. The first one is a $12.66 \mathrm{kV}, 33$ node network, which consists of 5 normally open switches and 32 normally closed switches. The normally open tie-switches are 33, 34, 35, 36 and 37, and the normally closed switches are 1 to 32 . The line data and load data of this network are given in [19], and the total real and reactive power loads on the network are $3715 \mathrm{~kW}$ and 2300 $\mathrm{kVar}$, respectively. The second test network is a $12.66 \mathrm{kV}, 69$ node network comprising of 5 tie-loops. The network data of the initial configuration is available in [20]. The total active and reactive network loads are $3802.19 \mathrm{~kW}$ and 2694.60 $\mathrm{kVar}$, respectively.

The reconfiguration problem for this case is solved by GA and PSO in addition to solving by BRM. In this regard, the same set of decision variables and fitness/cost function, involved in the BRM, are used to develop the GA and PSO approaches. The results of 33 node network, containing details of openswitches, NVD and lowest voltage magnitude (LVM), before and after reconfiguration are presented in Table-1. It is very clear from the results that the proposed BRM reduces the initial NVD of 1.8046 to 1.0668 , which leads to \%VP improvement (\%VPI) of $40.88 \%$, while the GA and PSO offer $39.45 \%$ and $39.79 \%$ of \%VPI. The LVM of the BRM is better than those of the GA and PSO. The VP for 33 node system before and after reconfiguration is pictorially depicted in Fig.A.1. It is seen from the figure that there is significant improvement in the VP after reconfiguration. It increases the LVM seen in the network from 0.9038 to 0.9327 per unit. 
Table.1 Summary of Results for 33 node network

\begin{tabular}{|c|c|c|c|}
\hline & Open-switches & NVD & LVM \\
\hline Initial Configuration & $33,34,35,36,37$ & 1.8046 & 0.9038 \\
\hline BRM & $7,14,9,17,28$ & 1.0668 & 0.9327 \\
\hline GA & $33,34,10,17,28$ & 1.0927 & 0.9313 \\
\hline PSO & $33,34,11,36,28$ & 1.0865 & 0.9319 \\
\hline
\end{tabular}

The details of open-switches, NVD and LVM before and after reconfiguration for 69-node network are presented in Table2. It is obvious from the results that the proposed BRM is able to reduce the initial NVD of 1.8370 to 0.8273 , which is leads to \%VPI of $54.96 \%$, while the GA and PSO offer $49.98 \%$ and $54.16 \%$ of \%VPI. The LVM of all the three methods is found to lie in between the lower and upper limits. The VP for 69 node system before and after reconfiguration of the proposed BRM is pictorially depicted in Fig. A.2. It can be observed from the figure that there is considerable improvement in the VP after reconfiguration.

Table.2 Summary of Results for 69 node network

\begin{tabular}{|c|c|c|c|}
\hline & Open -switches & NVD & LVM \\
\hline Initial Configuration & $69,70,71,72,73$ & 1.8370 & 0.9092 \\
\hline BRM & $14,57,64,69,70$ & 0.8273 & 0.9382 \\
\hline GA & $69,14,70,56,61$ & 0.9189 & 0.9495 \\
\hline PSO & $69,14,70,58,62$ & 0.8421 & 0.9483 \\
\hline
\end{tabular}

It is very clear from these results that the proposed BRM offers a better configuration that reduces the NVD for both the test networks and offers a good VP.

\section{CONCLUSIONS}

A simple BBO based reconfiguration scheme for minimization of NVD of radial distribution networks has been developed. This method uses a simple distribution power flow taking into the status of open-switches for calculating the NVD. It uses a simple mechanism for checking the radial nature of the network. The results on 33 and 69 node networks have clearly indicated that the proposed method is able to offer a better VP without any additional infrastructural cost. The algorithm is suitable for practical implementation on networks of any size. The method can be further modified to enhance voltage stability of the distribution networks.

\section{ACKNOWLEDGMENTS}

The authors gratefully acknowledge the authorities of Annamalai University for the facilities offered to carry out this work.

\section{REFERENCES}

[1]. Merlin A and Back H. (1975). Search for a minimal loss operating spanning tree configuration for an urban power distribution system, Proc of the power systems computation conf (PSCC): 1-18.

[2]. H. M. Khodr and J. Martinez-Crespo. (2009). Integral methodology for distribution systems reconfiguration based on optimal power flow using benders decomposition technique, IET Gen. Trans. \& Dist., 3(6): 521-534.

[3]. R. A. Jabr, R. Singh and B. C. Pal. (2012). Minimum loss network reconfiguration using mixed-integer convex programming, IEEE Trans on Power Systems, 27 (2): 1106-1115.

[4]. J. A. Taylor and F. S. Hover. (2012). Convex models of distribution system reconfiguration, IEEE Trans. On Power Systems, 27(3): 1407-1413.

[5]. Civanlar S, Grainger J and Yin H, Lee S .(1988). Distribution feeder reconfiguration for loss reduction, IEEE Trans Power Delivery, 3(3): 1217-1223.

[6]. Goswami S and Basu S. (1992). A new algorithm for the reconfiguration of distribution feeders for loss minimization, IEEE Trans Power Delivery, 7(3):14821491.

[7]. Shirmohammadi D and Hong H. (1989). Reconfiguration of electric distribution networks for resistive line loss reduction, IEEE Trans Power Delivery, 4(2):1492-1498.

[8]. A.Y. Abdelaziz, R. A. Osama and S. M. El-Khodary. (2012). Reconfiguration of distribution systems for loss reduction using the hyper-cube ant colony optimization algorithm, IET Gen. Trans. \& Dist., 6(2): 176-187.

[9]. K. Sathish Kumar and T. Jayabarathi. (2012). Power system reconfiguration and loss minimization for distribution systems using Bacterial Foraging optimization algorithm, Int. J. Electr. Power Energy Syst, 36(1): 13-17.

[10]. M. R. Andervazh, J. Olamaei and M. R. Haghifam. (2013). Adaptive multi-objective distribution network reconfiguration using multi-objective discrete Particles Swarm Optimisation algorithm and graph theory, IET Gen. Trans. \& Dist., 7(12): 1367-1382.

[11]. L. W. de Oliveira, E. J. de Oliveira, F. V. Gomes, I. C. Silva Jr, A. L. M. Marcato and P. V. C. Resende. (2014). Artificial Immune Systems applied to the reconfiguration of electrical power distribution networks for energy loss minimization, Int. J. Electr. Power Energy Syst., 56: 64-74.

[12]. S. H. Mirhoseini, S. M. Hosseini, M. Ghanbari and M. Ahmadi. (2014). A new improved adaptive imperialist competitive algorithm to solve the reconfiguration problem of distribution systems for loss reduction and voltage profile improvement, Int. J. Electr. Power Energy Syst., 55: 128-143.

[13]. N. Gupta, A. Swarnkar and K. R. Niazi. (2014). Distribution network reconfiguration for power quality and reliability improvement using Genetic Algorithms, Int. J. Electr. Power Energy Syst., 54: 664-671.

[14]. D Simon. (2008). Biogeography-based optimization, IEEE Trans on Evolutionary Computation, 12(6): 702 713.

[15]. R Rarick, D Simon, F Villaseca and B Vyakaranam. (2009). Biogeography-based optimization and the solution of the power flow problem, IEEE International Conference on Systems, Man, and Cybernetics, San Antonio, SMC: 1003-1008.

[16]. A Bhattacharya and K.P Chattopadhyay. (2010). Solution of optimal reactive power flow using biogeography-based optimization, Int Journal of Electrical and Electronics Engineering, 4(8): 568-576.

[17]. A.Bhattacharya and K.P Chattopadhyay. (2011). Application of biogeography-based optimization to solve different optimal power flow problems, IET Proc Gener., Transm \& Distrib, 5(1): 70-80.

[18]. S.Rajasomashekar and P Aravindhababu. (2012). Biogeography-based optimization technique for best compromise solution of economic emission dispatch, Swarm and Evolutionary Computations, dx.doi.org/10.1016/j.swevo.2012.06.001. 
[19]. Baran M and Wu F. (1989). Network reconfiguration in distribution systems for loss reduction and load balancing, IEEE Trans on Power Delivery, 4(2): 14011407.

[20].Kashem M, Ganapathy V and Jasmon G (2001) A geometrical approach for network reconfiguration based loss minimization in distribution systems, Int $\mathrm{J}$ Elect Power Energy Syst, 23(4): 295-304.

\section{APPENDIX}

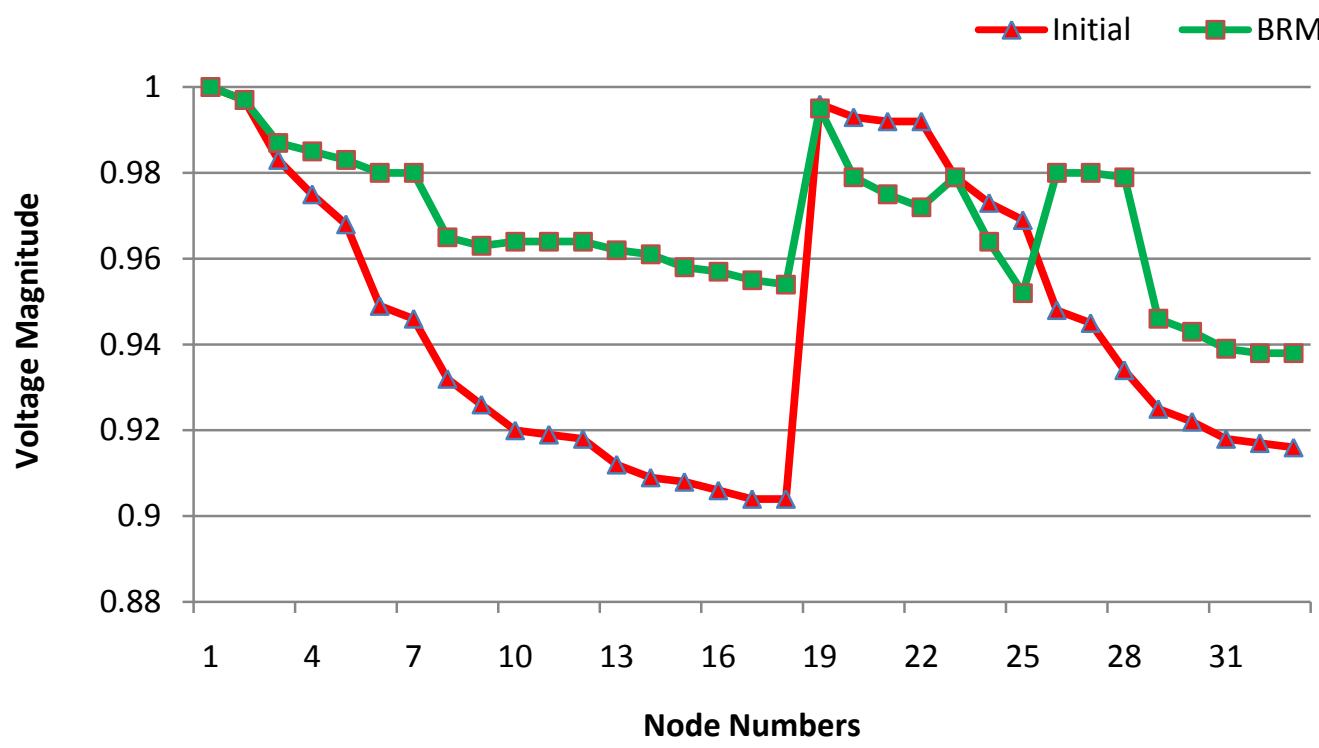

Fig. A.1 VP of 33 node network

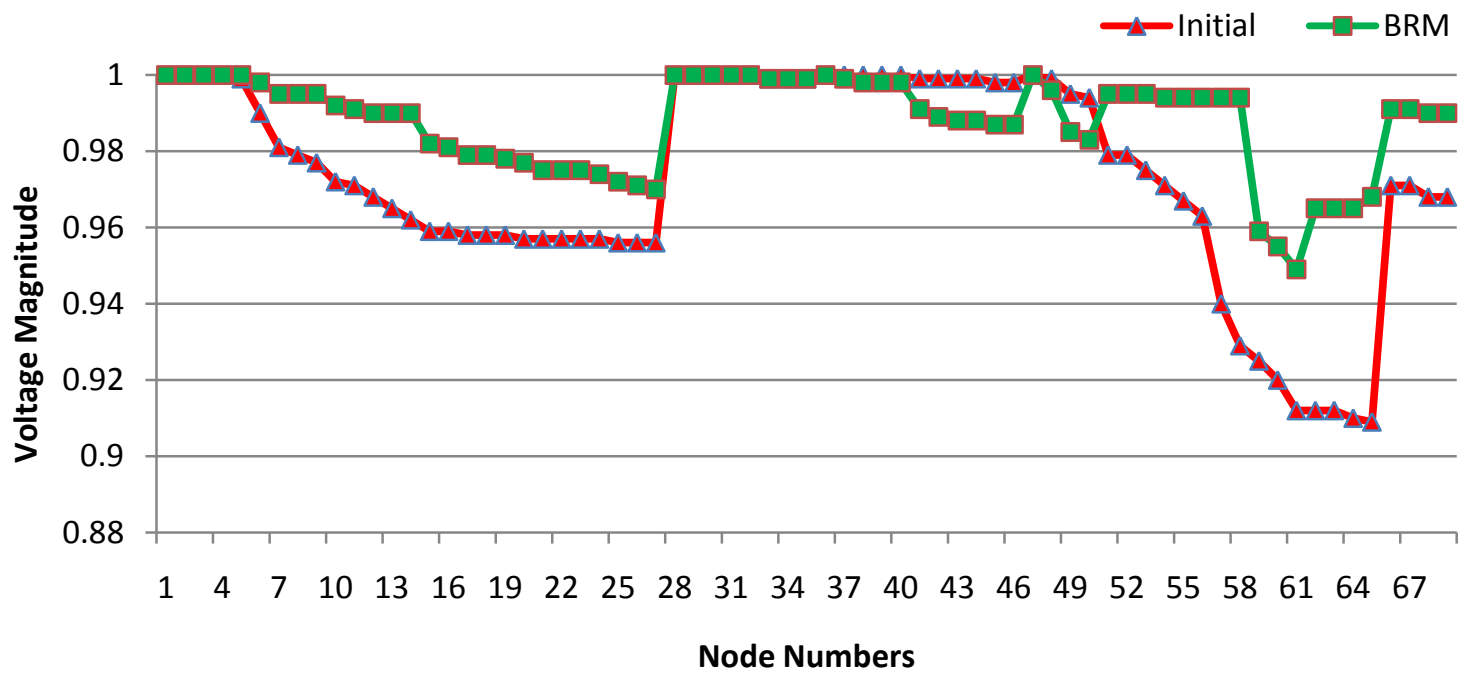

Fig. A.2 VP of 69 node network 